\title{
Commentary on High-Performance Computing and Statistical Analysis: From large-scale data analysis to smoothing functions
}

\author{
MARK L. DAVISON \\ University of Minnesota, Minneapolis, Minnesota
}

\begin{abstract}
High-performance computing becomes essential to statistical analysis when the database is massive or the number of computations per data element is large. Albert F. Anderson (1997) discusses the application of high-performance computing to massive databases; J. O. Ramsay's (Ramsay, Heckman, \& Silverman, 1997) estimation problems potentially require large numbers of computations per data element.
\end{abstract}

The databases to which Albert F. Anderson (1997) refers contain millions of records and occupy disk storage measured in gigabytes. The user sits at a remote site and requests computations through a graphical user interface over the internet from parallel processors which speed up data analysis by dividing computations between processors such that the several processors are operating simultaneously, each on its own portion of the data. For those of us over 40 , this is a return to batch processing and central computing. But with this configuration, computations that formerly took hours, weeks, or even months can be reduced to seconds.

Parallel processing requires software which can parse computations to separate processors. Not all computational problems will lend themselves to parsing as fully as the ones described by Anderson. When later computations are conditional on the results of earlier ones, as in many iterative algorithms, the computations must be performed sequentially rather than in parallel.

Anderson's parallel processing will make it possible for social and behavioral scientists to use data sets that are currently unrealistically impractical. Anderson mentions Chinese census data containing 100 million records and concatenated U.S. census data from 1860 to 1990 . Members of the audience mentioned others, such as data from financial markets around the world and extending back in time. Because of the system's speed, researchers can ask more computationally complex questions. The swift turnaround and interactive character will allow us to refine our analysis, reanalyzing as needed to improve the results.

Most of us are concerned about the estimation of parameters (or parameter vectors), whereas $\mathrm{J}$. O. Ramsay (Ramsay, Heckman, \& Silverman, 1997) is concerned with

Requests for reprints should be sent to M. L. Davison, Department of Educational Psychology, University of Minnesota, Minneapolis, MN 55455 (e-mail: mld@maroon.tc.umn.edu). the estimation of functions. His major examples involve functions that approximate growth or change over time.

He calls his approach "smart" smoothing because it incorporates a priori information about the form of the function. This is in contrast to "dumb" smoothing, which, roughly speaking, simply minimizes (or maximizes) a loss function without incorporating prior information about the form of the function.

Ramsay accomplishes smart smoothing by first expressing the a priori functional form as a differential equation. He then creates a function to be minimized (or maximized) that consists of a conventional loss function (least squares in his examples) and a penalty function expressed in terms of the differential equation.

An impressive example involves quarterly Swedish gross domestic product data from 1980 to 1994 . His smartly smoothed function reproduces the general upward trend over the 24-year period, as well as the oscillating seasonal trend within years. The "dumb" polynomial smoother yields a function that reproduces the yearly upward trend but not the oscillating seasonal trend within years.

Several points about Ramsay's approach are worth noting. First, it requires differentiable functions. Second, in the absence of good information about the form of the function, simpler polynomial smoothing seems preferred. Third, smart smoothers are only as "smart" as their user, since they require good a priori information about the form of the function. Finally, as the data become more sparse, using a smart smoother offers more advantages, because the sparse data alone may not be sufficient to define the appropriate functional form for the data.

Ironically, Ramsay and his coworkers have been working to reduce the computational intensity of smoothing with model-based penalties, and hence the need for highperformance computing - at least for the models illustrated by Ramsay. In the ensuing discussion, however, it was pointed out that the functions in the talk were deterministic rather than stochastic, and that differential Mar- 
tingale functions offer a basis for a stochastic rendering of the deterministic models. Implementation of fully stochastic models will almost certainly be computationally more intensive.

From these papers, it is clear that high-performance computing will make it possible to analyze larger databases and to use computationally intensive techniques, such as bootstrapping and smart smoothing. To realize these possibilities, however, statistical software must be redesigned to take advantage of parallel processing; user interfaces must be developed to make the processing ac- cessible to working social scientists; and working social scientists need more access to the hardware which supports the redesigned software.

\section{REFERENCES}

ANDERSon, A. F. (1997). Application of high-performance computing to the management of social science and demographic data. Behavior Research Methods, Instruments, \& Computers, 29, 86-98.

Ramsay, J. O., Heckman, N., \& Silverman, B. W. (1997). Spline smoothing with model-based penalties. Behavior Research Methods, Instruments, \& Computers, 29, 99-106. 